Humphrey, J. H. \& Lightbown, J. W. (1952). J. gen. Microbiol. 7, 129-143

\title{
A General Theory for Plate Assay of Antibiotics with some Practical Applications
}

\author{
BY J. H. HUMPHREY AND J. W. LIGHTBOWN \\ National Institute for Medical Research, Mill Hill, London, N.W. 7
}

\begin{abstract}
SUMMARY: The distribution of antibiotic (or other substance) in the agar around a container or around a hole in a punch-plate can be expressed theoretically by an equation involving: the initial quantity of antibiotic, the depth of the agar layer, the diffusion constant, the concentration at a given distance from the container, and the time of diffusion. The validity of the equation was confirmed by measurement of the diffusion constants of penicillin, streptomycin and aureomycin, and of the critical concentration of these substances required to inhibit test organisms, followed by the use of the values so obtained to predict the sizes of the inhibition zones produced experimentally by these antibiotics after varying periods of diffusion.

The theory predicts that the square of the inhibition zone diameter will be proportional to the logarithm of the antibiotic concentration. This relationship was found to hold, when accurate assays were made, for a number of antibiotics but not for penicillin when tested with Bacillus subtilis. The most important factor determining the slope of the dose-response curve under given conditions is the diffusion constant of the antibiotic. The slope can, however, be increased by prolonging the time allowed for diffiusion.

Particular factors which affected both the sharpness of the zone edge and the nature of the dose-response curve were production of small amounts of penicillinase by strains of $\boldsymbol{B}$. subtilis used for penicillin assay, and uptake of streptomycin by organisms used for streptomycin assay. Measurements of adsorption of streptomycin by $\boldsymbol{B}$. subtilis, B. pumilus and by Staphylococcus aureus were made, and were shown to fit equations of the Freundlich isotherm type.
\end{abstract}

Although very good plate assay methods are available for penicillin and streptomycin, those described for the newer antibiotics are less satisfactory owing to a tendency for the zone edges to be poorly defined and to shift their position as incubation of the plates is continued. Unless a valid theoretical treatment of the nature of diffusion of an antibiotic from the cup into the surrounding agar gel is available, attempts to investigate the factors governing the nature of the zone edge and the slope of the dose-response curve for new antibiotics will be empirical and time-consuming. The only theoretical treatment of this problem of which we were aware was that given by Cooper \& Woodman (1946), who discussed diffusion of antiseptics and of penicillin from a Heatley cup into agar, and claimed good agreement between predicted zone diameters and those which they observed in practice. They used, however, a formula derived from considerations of linear, not radial, diffusion, and assumed that the concentration of antibiotic in the cup was constant during the first $8 \mathrm{hr}$. These assumptions do not hold in plate assays as often performed, and we decided therefore to investigate the theoretical treatment from first principles. With the aid of certain assumptions, which are discussed below, a theoretical expression was derived, differing somewhat from that given by 
Cooper \& Woodman; its validity was tested in various ways. These tests revealed that in penicillin and streptomycin assays, under certain conditions, there is a sudden fall in concentration of antibiotic at the zone boundary, which results in a very sharp edge. Tests were also made to discover the reason for this boundary effect.

\section{METHODS}

Fish spine beads, size no. 2 (obtainable from Messrs Taylor, Tunnicliffe and Co., 125 High Holborn, London, W.C. 1) were used (Stewart \& Thorpe (to be published)). These beads are very uniform, and may be assumed to hold on the average $0.025 \mathrm{ml}$.

Assay trays. Large flat trays, $10 \times 10 \mathrm{in}$. accommodating 64 beads were used (Kantorowicz, 1951).

Organisms. Spores of B. subtilis, I.C.I. strain (NCTC 8236) and B. pumilus Mill Hill strain (NCTC 8241) were used for most assays. Suspensions were incorporated into molten agar at $60-70^{\circ}$, at a density of approximately $3 \times 10^{7}$ and $6 \times 10^{7}$ viable spores $/ \mathrm{ml}$. of medium respectively.

For certain experiments suspensions of Staphylococcus aureus (NCTC 7447) or of Sarcina lutea (NCTC 8340) were used. Suspensions were made with a density equivalent to Brown's tube no. 6 (Burroughs Wellcome Ltd.) and to ten times this strength respectively, and were incorporated into molten agar at $45^{\circ}$ at a concentration of $1 \%(\mathrm{v} / \mathrm{v})$.

The strains used had been subjected to several single colony isolations and were stored freeze-dried, except for the $B$. subtilis and B. pumilus strains which were stored at $2^{\circ}$ as spore suspensions.

Media. Experiments with penicillin and aureomycin were performed with a Lemco Marmite peptone agar $\mathrm{pH} 7$ when $B$. subtilis I.C.I. strain was used, and with the enriched seed layer medium prescribed by the Food and Drugs Administration of the U.S. Federal Security Agency (1951) when other organisms were used.

Experiments with streptomycin and dihydrostreptomycin were carried out in nutrient agar containing peptone, beef extract and yeast extract $\mathrm{pH} \mathbf{7 \cdot 8}$.

Difco agar $2 \%$ was used in all media.

Zone diameters were measured on a screen after $\times 10$ magnification. Under the conditions of our assays the coefficient of variation of the diameter of a single zone was repeatedly found to be approximately $1 \%$.

Diffusion constants. These were measured by the method of Friedman \& Kraemer (1930). In this method a layer of agar gel is formed in a cylindrical container, and an equal volume of liquid is placed above it. The liquid layer is stirred continuously. Initially the substance whose diffusion constant is to be measured is either wholly in the agar layer or wholly in the liquid layer. By measurement of the change in concentration in the liquid layer, and the use of a theoretical expression derived by March \& Weaver (1928) for this system, the diffusion constant can be obtained. It should be noted that the theoretical expression quoted by Friedman \& Kraemer is wrong (although the curve which they give is correct), and the proper equation relating $v$, the fraction 
diffused across the boundary, and the diffusion constant $k$ is as follows (taken from March \& Weaver):

$$
v=\frac{1}{2}-\Sigma\left(\pi^{2} B i / 4 Z i^{2}\right) \mathrm{e}^{-Z i^{2} T}
$$

where $T=k t / a^{2}$. In this expression $a=$ depth of agar layer, $k=$ diffusion constant, $t=$ time, $Z$ is given by the roots of $\tan Z+Z=0$. $B$ is a function whose values are $B_{0}=0.545, B_{1}=0.748, B_{2}=0.789, B_{3}=0.799, B_{4}$ and higher terms $=0 \cdot 810\left(=8 / \pi^{2}\right)$.

This equation, expanded to 10 terms, becomes

$$
\begin{aligned}
& v=\frac{1}{2}-\left[0.327 \mathrm{e}^{-4 \cdot 117 T}+0 \cdot 0766 \mathrm{e}^{-24 \cdot 14 T}+0.0306 \mathrm{e}^{-63 \cdot 68 T}+0.016 \mathrm{e}^{-123 T}+0.01 \mathrm{e}^{-200 T}\right. \\
& \left.+0.0067 \mathrm{e}^{-300 T}+0.0048 \mathrm{e}^{-418 T}+0.0036 \mathrm{e}^{-556 T}+0.00279 \mathrm{e}^{-716 T}+0.00115 \mathrm{e}^{-886 T}+\ldots\right] .
\end{aligned}
$$

By the use of this expression a curve relating $v$ and $T$ can be drawn, from which the diffusion constant can be derived for values of $v>0.05$. (If the expression is to be applied for lower values of $v$ account must be taken of higher terms of the expansion.)

In our experiments the diffusion was measured under conditions as close as possible to those obtaining in the assay plates. The agar layer was made from nutrient agar which had been allowed to attain equilibrium with the nutrient broth used for the overlying liquid layer, by fragmentation of the agar, followed by 3 days contact at $2^{\circ}$. In the case of penicillin and dihydrostreptomycin the concentration in the small samples taken from the liquid layer was measured by an accurate biological method. In the case of aureomycin the concentration was measured colorimetrically, for which purpose concentrations of the order of $100-500 \mu \mathrm{g} . / \mathrm{ml}$. were required. These concentrations are greater than can be obtained in nutrient broth and therefore $1 \% \mathbf{K H}_{2} \mathbf{P O}_{4}$ was used in both layers. Control experiments were run to allow for possible inactivation of the antibiotics during the course of the experiment.

In order to ensure adhesion of the agar to the cylinder wall, the latter was coated with a layer of 'Perspex'.

\section{EXPERIMENTAL}

\section{Theory of diffusion}

All experiments were performed with cups consisting of the 'fish spine' insulator beads which are filled by capillarity, and whose effect is to place a uniform large drop on a uniform small area of the agar surface. Such a bead is shown in Fig. 1, which also shows the manner in which the contained liquid enters the agar layer. By the use of isotonic solutions of dyes we found that under average conditions a bead was empty in 2-3 hr., after which further spread occurred by simple diffusion. An approximate theoretical treatment can be derived on the assumption that a known quantity of antibiotic is placed in the agar at zero time in the form of an infinitely thin pencil, and that radial diffusion takes place from the pencil. Although this assumption is clearly not correct during the first $2 \mathrm{hr}$., it becomes increasingly true at later stages, and permits at least an approximate prediction of the behaviour of the diffusing substance. 
We are indebted to $\mathrm{Mr} \mathbf{A}$. G. Liddiard of the Department of Theoretical Physics, King's College, London, for deriving the following expression which describes the theoretical behaviour of such a system:

$$
\sigma=\frac{M}{4 \pi h D t} \mathrm{e}^{-r^{2} / 4 D t},
$$

where $\sigma=$ concentration of substance at a distance $r$ from the centre, $M=$ total quantity of dissolved substance, $h=$ depth of agar layer, $\boldsymbol{D}=$ diffusion constant, $t=$ time from start.

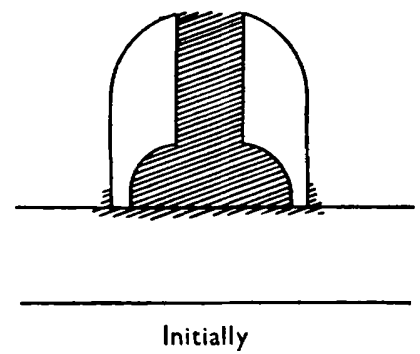

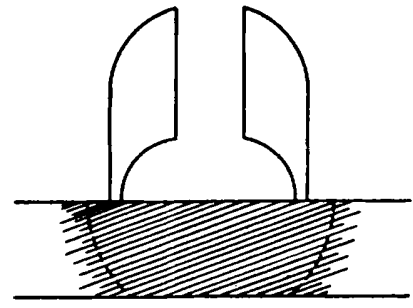

After 2-3 hr.

Fig. 1. Diagram illustrating movement of solution from a fish spine bead into the agar layer.

We found, after this work was completed, that Vesterdal (1947) had used a similar expression to predict the diffusion of penicillin out of the holes in a 'punch' plate, although he did not prove its validity in practice. When Heatley cups or dry paper disks are used, however, the expression might require modification.

The expression can be applied to the prediction of the behaviour of inhibition zones in antibiotic assays on the reasonable assumption that the zone edge appears where the concentration of antibiotic has a particular critical value at a definite time after beginning incubation. This period of time is the 'critical time', and will in general depend upon the growth characteristics of the test organism under the conditions of the assay and in certain circumstances upon the time interval between the appearance of the zone edge and its measurement.

\section{Test of validity of the theoretical expression}

The validity of the theory and the assumption made can be tested by attempting to deduce from it the zone diameters to be expected for different antibiotics after allowing various periods of diffusion before incubation. The theoretical expression (2) can be rewritten in the form:

$$
r^{2}=9 \cdot 21 D t(\log M-\log 4 \pi h D t \sigma),
$$

from which $r$, the radius of the zone, may be evaluated if the other terms are known. The values of $M$ and $h$ can be fixed at will.

The 'critical' concentrations $\sigma$ was determined experimentally by measuring the minimum inhibitory concentration of antibiotic in pour plates. A layer of agar medium seeded with the test organism was poured in a series of Petri dishes. When the agar was set, half was removed from each plate, and the 
corresponding spaces were filled by volumes of seeded agar identical in all respects with that removed, except that they contained graded concentrations of the antibiotic under test. The plates were incubated under conditions resembling, as far as possible, those used in our assays, and the growth in the 2 halves was compared. In the case of penicillin and streptomycin a fairly sharp limiting concentration could be distinguished, above which growth was almost entirely prevented. With aureomycin, however, although after $8 \mathrm{hr}$. incubation a sharp end-point could be distinguished, after $24 \mathrm{hr}$. incubation growth had occurred, albeit of diminishing intensity, in a number of higher concentrations. This was interpreted as being due to instability of aureomycin in nutrient agar at $\mathbf{3 5}^{\circ}$, with consequent loss of its bacteriostatic effect. The results of our determinations are given in Table 1 .

Table 1. Minimum inhibiting concentrations of antibiotics in agar plates seeded with test organisms

\begin{tabular}{|c|c|c|c|c|}
\hline & Temp. & & $\begin{array}{c}\text { Minimum } \\
\text { inhibiting } \\
\text { concentrations }\end{array}$ & $\begin{array}{r}\text { Time } \\
\text { (hr.) }\end{array}$ \\
\hline Penicillin & $\begin{array}{l}\mathbf{3 8}^{\circ} \\
\mathbf{2 8}^{\circ}\end{array}$ & $\begin{array}{l}\text { B. subtilis I.C.I. } \\
\text { Sarcina lutea }\end{array}$ & $\begin{array}{l}0.018 \mathrm{u} . / \mathrm{ml} \text {. } \\
0.008 \mathrm{u} . / \mathrm{ml} \text {. }\end{array}$ & $\begin{array}{c}8-24 \\
16\end{array}$ \\
\hline Streptomycin & $38^{\circ}$ & B. subtilis I.C.I. & $0.03 \mathrm{u} . / \mathrm{ml}$ & 8-24 \\
\hline Dihydrostreptomycin & $38^{\circ}$ & B. subtilis & $0.06 \mathrm{u} . / \mathrm{ml}$ & 8-24 \\
\hline Aureomycin $\mathbf{H C l}$ & $\mathbf{3 5}^{\circ}$ & Staph. aureus & $\begin{array}{l}0 \cdot 04 \mu \mathrm{g} . / \mathrm{ml} \text {. } \\
\text { c. } 0 \cdot 10 \mu \mathrm{g} . / \mathrm{ml} .\end{array}$ & $\begin{array}{r}8 \\
24\end{array}$ \\
\hline
\end{tabular}

The diffusion constants $(D)$ were measured as described under Methods, and some results are recorded in Table 2 . Most measurements were made at $4^{\circ}$ because in subsequent experiments diffusion in the cold proved to be necessary to prevent growth of the test organism and to minimize inactivation of such antibiotics as are unstable under assay conditions. Diffusion constants are much affected by changes in the viscosity of water, and hence by changes of temperature, but corrections for this can be applied on the basis of tables available in International Critical Tables (1929).

Table 2. Diffusion constants in $2 \%$ agar

$\begin{array}{lcc} & & \begin{array}{r}\text { agar } \\ \text { Diffusion } \\ \text { constant } \\ \left(\mathrm{cm} .{ }^{2} / \mathrm{hr} .\right)\end{array} \\ \text { Penicillin } & \text { 'Temp. } & 0 \cdot 011 \\ \text { Penicillin } & 20^{\circ} & 0 \cdot 016 \\ \text { Penicillin } & 37^{\circ *} & 0 \cdot 0066 \\ \text { Aureomycin } & 4^{\circ *} & \mathbf{0 \cdot 0 0 7 5} \\ \text { Dihydrostreptomycin } & 4^{\circ} & 0 \cdot 0053\end{array}$

* Calculated by comparison with tables of diffusion constants given in International Critical Tables (1929).

The critical time (i.e. the time after incubation is begun at which the zone size and edge are determined) was not easy to determine in practice. The problem was approached in two ways. In the first, which was used by Cooper \& Woodman (1946), assay plates were poured containing seeded agar, and beads containing a constant amount of antibiotic solution were placed on them 
at varying intervals of time after incubation was begun. It was argued that beads placed after the critical time should show no inhibition zones. The drawback to this method lay in deciding whether to take account of small zones of inhibition which appeared even after general growth had occurred, and were associated with such high antibiotic concentrations that they were probably due to lysis. The second method consisted in placing many beads containing a constant concentration of antibiotic upon an assay plate. The plate was incubated at $37^{\circ}$, and from it at varying intervals of time, beads were removed, together with the agar immediately below and around them, by means of a cork borer. After the critical time removal of the bead may be expected not to affect the zone size. Estimates of the critical time obtained by the second method have always been about $1 \mathrm{hr}$. shorter than those by the first method, and for practical purposes we have taken a mean value. Since the concept of a sharp critical time is somewhat abstract, it is doubtful whether greater precision could be obtained.

With the values obtained it was possible to predict theoretically the zone diameters when antibiotics were allowed to diffuse for varying periods of time into seeded agar plates in the cold (i.e. without growth of the test organism) before incubation was begun. In order to permit sufficient replicate beads for accurate measurement at each time interval, it was necessary to use two assay plates in a single experiment. These were prepared as alike as possible, and one of the sets of replicate beads was repeated on each plate, as a check to ensure satisfactory duplication. In Fig. 2 are shown the experimental zone diameters observed at two levels of aureomycin concentration in relation to those predicted theoretically using the value for the diffusion constant obtained at $4^{\circ}$. Although failure to correct for the increased diffusion constant during the incubation period entails some error in the theoretical curves, it will not be great since the incubation period was short compared with the total period allowed for diffusion. It will be seen that the experimental points lie fairly close to the predicted curves, although a better fit is obtained with a slightly lower value for the critical concentration than that recorded in Table 1.

Fig. 3 shows a similar experiment, using penicillin with $B$. subtilis as test organism, conducted throughout at $37^{\circ}$. Because growth occurred throughout the experiment at this temperature the observation period was limited to $\mathbf{3} \frac{1}{4} \mathrm{hr}$. By using short time intervals and many replicate beads we obtained enough experimental points to show that under these conditions also agreement with the theory was remarkably good, except when the period of diffusion was brief. Since in this part of the curve the time interval between placing beads on the agar and the appearance of inhibition zones was too short to allow complete entry of the fluid into the agar, the conditions assumed in the theory did not apply, and the aberrant points can be discounted.

\section{Consequences of the theory}

Regarding the theory as substantially valid we can deduce some practical consequences which will apply not only to inhibition zones produced by antibiotics but also to exhibition zones produced by growth factors. 
(i) Fig. 4 shows some distribution curves of antibiotic in the agar around a bead, calculated for varying intervals of time. The figure in question relates to penicillin, but the general form of the curves applies to other substances, and it emphasizes how greatly the concentration at the centre exceeds that at the boundary. In the case of the diffusion assay of growth factors it is clear that there is little likelihood of organisms which grow inside the zone exhausting the growth factor to any significant extent.

(ii) From equation (3) it is apparent that under any given set of assay conditions $r^{2}$ should be proportional to $\log \boldsymbol{M}$.

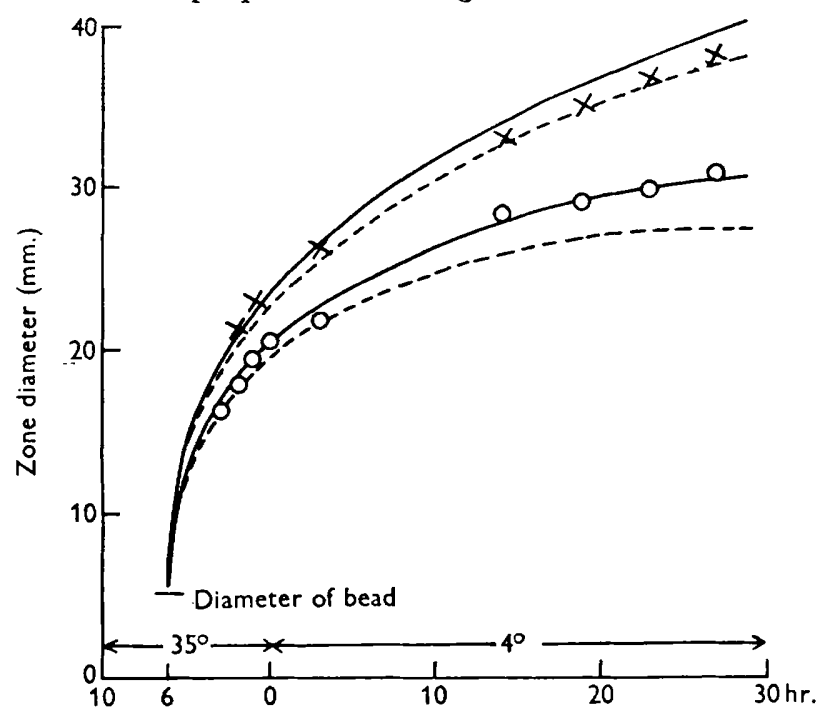

Fig. 2. Zone diameters observed after varying periods of diffusion in the cold followed by incubation at $\mathbf{3 5}^{\circ}$, compared with diameters predicted theoretically. Antibiotic: aureomycin; test organism: Staph. aureus. $\times \times$ and 00 are experimental points for beads containing solutions of 25 and $6 \cdot 25 \mu \mathrm{g} . / \mathrm{ml}$. respectively. Each point is the mean of 5 diameters, which were read after $8 \mathrm{hr}$. incubation. The theoretical curves are drawn from the expression

$$
r^{2}=9 \cdot 21 D t(\log M-\log 4 \pi h D t \sigma),
$$

where $D=0.0075 \mathrm{~cm} .{ }^{2} / \mathrm{hr} ., \quad h=0.4 \mathrm{~cm}$, and $M=0.625$ and $0.156 \mu \mathrm{g}$. when $\sigma=0.025 \mu \mathrm{g} . / \mathrm{ml}$; - - - , curves when $\sigma=0.035 \mu \mathrm{g} . / \mathrm{ml}$.

There has been much discussion as to whether the relationship between the log. of the antibiotic concentration and zone diameter is linear or whether a square term is involved. The general, although not the universally agreed, conclusion is that in penicillin assays the relationship is linear. It must be borne in mind, however, that a significant departure from a linear relationship will be noticed only if an assay is very accurate (in the sense that the coefficient of variation of a single zone is small and many replicates are used) or if a wide range of concentrations of antibiotic is used. We have found that when very accurate assays were performed (using 64 replicates at each dose level) the logarithm of the antibiotic concentration was more nearly proportional to the square of the zone diameter than to the zone diameter in the following assays: streptomycin and dihydrostreptomycin with $B$. subtilis as test organism; 
aureomycin and terramycin with $B$. pumilus; penicillin with Staph. aureus. When penicillin was assayed with $B$. subtilis I.C.I. strain the zone diameter, and not its square, gave the best fit. A possible reason for this discrepancy, which may have wider application, is given below.

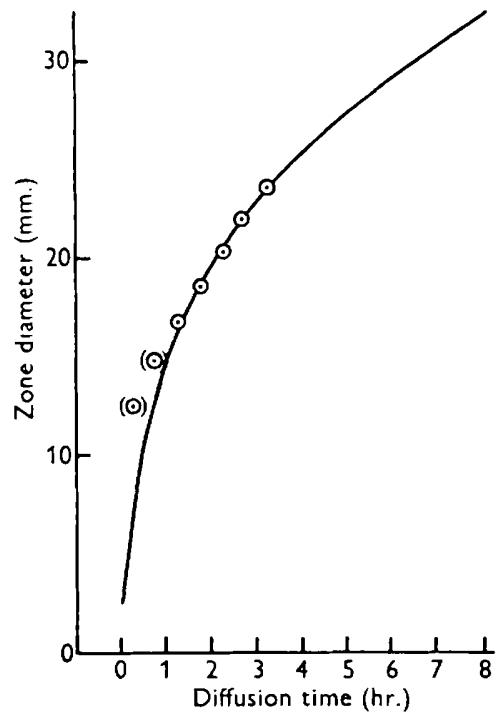

Fig. 3

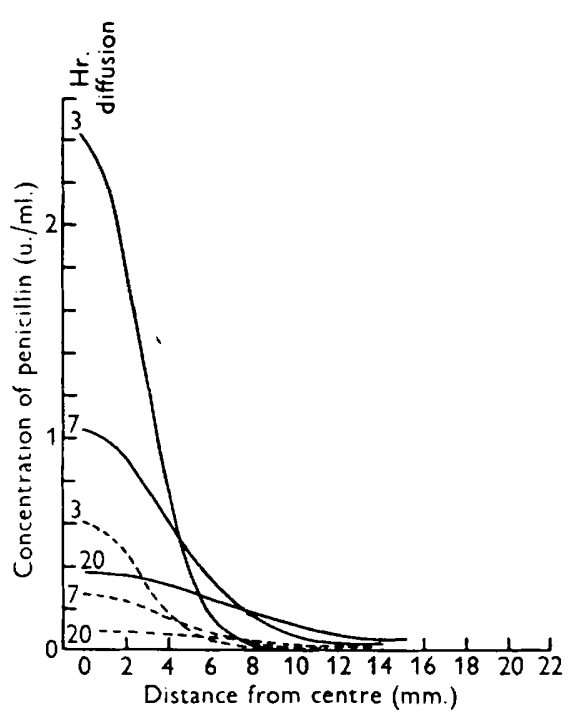

Fig. 4

Fig. 3. Zone diameters observed after varying periods of diffusion at $37^{\circ}$, compared with those predicted theoretically. Antibiotic: penicillin; test organism: $B$. subtilis. Each experimental point is the mean of 16 beads, containing a solution $16 \mathrm{u} . / \mathrm{ml}$. The theoretical curve was plotted from

$$
r^{2}=9 \cdot 21 D t(\log M-\log 4 \pi h D t \sigma),
$$

where $D=0.016 \mathrm{~cm} .{ }^{2} / \mathrm{hr} ., \quad M=0.4$ unit, $h=0.4 \mathrm{~cm} ., \sigma=0.02 \mathrm{u} . / \mathrm{ml}$.

Fig. 4. Curves illustrating the distribution of penicillin concentration in an agar layer depth $0.4 \mathrm{~cm}$. around fish spine beads containing penicillin solution, after 3,7 and $20 \mathrm{hr}$. diffusion at room temperature. __, bead with $16 \mathrm{u} . / \mathrm{ml}$.; - - - , bead with $4 \mathrm{u} . / \mathrm{ml}$. The critical concentration is $0.02 \mathrm{u} . / \mathrm{ml}$.

(iii) The sharpness of the edge of a zone, apart from effects due to lysis, will depend upon the range of antibiotic concentrations over which partial but not complete inhibition of growth occurs. Suppose that $\sigma_{2}$ is the critical concentration which permits no growth, and $\sigma_{1}$ the concentration which permits full growth of the test organism. Then over the range $\sigma_{1}$ to $\sigma_{2}$ a shading of growth will occur. Let $r_{1}$ and $r_{2}$ be the corresponding distances from the cup, so that the width of the edge is $r_{1}-r_{2}$. From the expression (3) it can be shown that

$$
r_{1}-r_{2}=\frac{9 \cdot 21 D t}{2 R} \log \left(\frac{\sigma_{2}}{\sigma_{1}}\right) \text {, }
$$

where $R$ is the mean of $r_{1}$ and $r_{2}$. If therefore the response of the organism to the antibiotic cannot be made homogeneous (e.g. by single colony selection), the effects on the zone edge will be minimized when the diffusion time is short and the zone diameter large. 
(iv) Factors governing the slope of the dosage-response curve can be evaluated as follows. If $d_{1}$ and $d_{2}$ are the zone diameters when the amounts of antibiotic in the cup are $M_{1}$ and $M_{2}$, it is readily shown that

i.e.

$$
d_{1}-d_{2}=\frac{9 \cdot 21 D t}{R}\left(\log M_{1}-\log M_{2}\right)
$$

$$
\frac{d_{1}-d_{2}}{\log M_{1}-\overline{\log } M_{2}}={ }^{\prime} \text { slope' }=-\frac{9 \cdot 21 D t}{R} \text {. }
$$

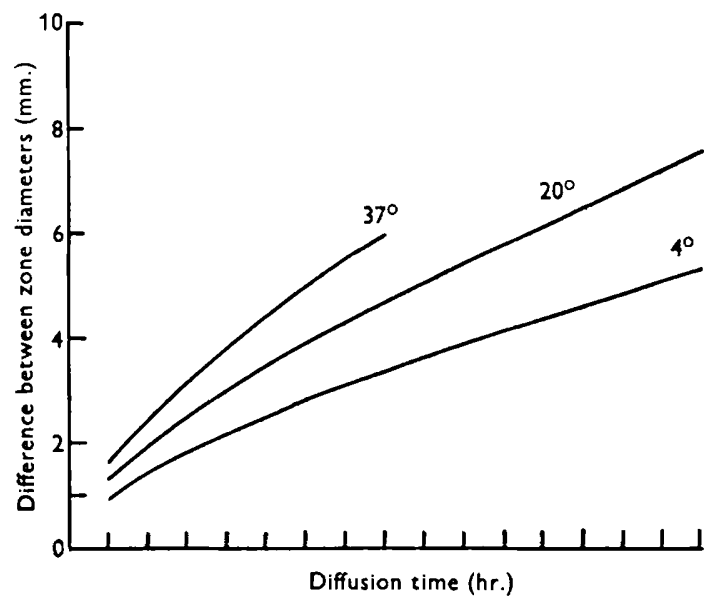

Fig. 5. Theoretical curves demonstrating the relationship between slope and diffusion time for a penicillin assay at levels of 16 and $4 \mathrm{u} . / \mathrm{ml}$. Curves are shown for three different temperatures of diffusion. No correction of the 4 and $20^{\circ}$ curves is made for subsequent incubation at $37^{\circ}$. The values used for $D$ are given in Table 2.

From (4) it appears that the slope will vary directly with diffusion time, and inversely with the mean zone radius. It will also depend directly upon the diffusion constant. Two practical conclusions can be drawn. The first is that when assays of several antibiotics are preformed under standard conditions, and when, as occurs in practice, zone diameters are chosen for convenience to be about the same, the main factor governing the slope will be the diffusion constant. The commoner antibiotics all have similar diffusion constants, and the slopes in their assays will consequently also be similar.

The second conclusion is that the slope can be increased by prolonging the diffusion time and by keeping the antibiotic concentrations and therefore the zone diameters small. In practice, the value of such measures can be limited by loss of definition of the zone edge, for they are precisely opposite to the recommendations of $\S$ (iii). Fig. 5, however, illustrates the effect upon the slope of penicillin assays of the length of the diffusion time at different temperatures.

The special cases of penicillin and streptomycin assays

When penicillin assays are performed with the I.C.I. strain of $B$. subtilis, and streptomycin assays with $B$. subtilis or other Gram-positive organisms, the zone edges are characteristically very sharp; they do not shift with 
continued incubation; and contiguous zones do not distort one another. These observations suggested that there might be a sharp drop in antibiotic concentration at the zone edge, which might be due either to its destruction or to its removal by the organisms at that site. Investigations have shown such a drop to occur, although the mechanisms in the two cases are different and must be treated separately.

\section{Penicillin assays}

An experiment was devised to discover whether penicillin did in fact diffuse beyond the zone edge of $\boldsymbol{B}$. subtilis seeded plates. For this purpose plates were prepared which were seeded with a mixture of I.C.I. strain $B$. subtilis spores and of $S$. lutea. The sarcina is about three times as sensitive as the subtilis to penicillin, and the two organisms grow well in each other's presence. This
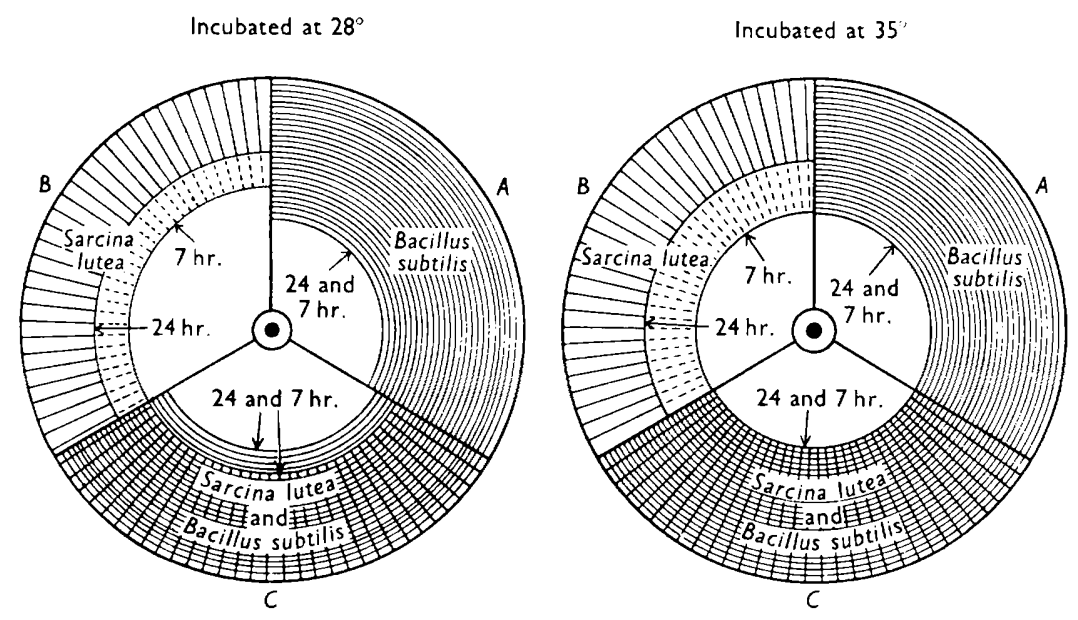

Fig. 6. Scale diagram of an experiment which illustrates the failure of penicillin to diffuse past zone edges formed by $B$. subtilis. Matched plates were poured with agar seeded with $B$. subtilis alone $(A)$, Sarcina lutea alone $(B)$, and a mixture of the two $(C)$. Beads containing $16 \mathrm{u} . / \mathrm{ml}$. penicillin were placed on each plate and the inhibition zones were measured at intervals during incubation at 28 or $35^{\circ}$. Sarcina alone gave a wide zone which increased with time owing to lysis. In the mixed plate at $28^{\circ}$ the Sarcina zone appeared first, but became fixed when the $B$. subtilis zone appeared inside it. At $\mathbf{3 5}^{\circ}$ growth of both organisms became apparent at the same time, and Sarcina appeared and remained present right up to the $B$. subtilis zone edge.

point was verified by examination of stained smears from the agar. Beads containing a fixed quantity of penicillin were placed upon plates seeded with the mixture of organisms, and upon control plates containing each of the organisms alone but in other respects identical. Sets of plates were incubated at 28 and $35^{\circ}$, since by varying the temperature it was possible to accelerate or retard the growth of $B$. subtilis relative to that of $S$. lutea. The plates were examined at intervals, and Fig. 6 illustrates diagrammatically the behaviour of the inhibition zones after 7 and $24 \mathrm{hr}$. The $S$. lutea zones shifted outwards as incubation continued, whereas the $B$. subtilis zones remained fixed. When both organisms grew together the $S$. lutea zones became fixed, and the size at which 
they were fixed depended upon the time at which significant growth of $B$. subtilis had occurred. The most probable explanation is that penicillin did not get past the $B$. subtilis zone edge.

Removal of penicillin by adsorption by the organisms might provide a possible explanation of such an effect, but calculations based on the data of Rowley, Cooper, Roberts \& Smith (1950) showed that the amount adsorbed would be far too small. We were therefore led to test for penicillinase production. In order that conditions of growth of the B. subtilis should be comparable to those obtaining in assays, plates seeded with spores were incubated under standard conditions. After $16 \mathrm{hr}$. the agar was removed and frozen solid in a closed container, after which it was allowed to thaw. The liquid formed was collected, and was sterilized by filtration through a Gradocol membrane. Aliquots of the liquid were incubated at $37^{\circ}$ in the presence of phosphate buffer $\mathrm{pH} ; \cdot 0$ for varying periods of time with varying amounts of penicillin. The anount of penicillin destroyed was estimated biologically. In a typical experiment with a filtrate from the I.C.I. strain the rate of inactivation of penicillin, present at an initial concentration of $16 \mathrm{u} . / \mathrm{ml}$., was $28 \mathrm{u} . / \mathrm{ml}$. filtrate per hour. The enzymic nature of this inactivation was confirmed by showing that the extent was proportional to the time of incubation. Such a low penicillinase activity would escape detection by most methods, but it is sufficient. nevertheless, to destroy penicillin at the zone boundary, as the following calculations show.

Consider an annular ring of agar $25 \mathrm{~mm}$. diam., of depth $4 \mathrm{~mm}$. and of width $0.05 \mathrm{~mm}$. This is approximately the size of the boundary area of a typical inhibition zone, and its volume is 0.015 c.c. When once the organisms have grown, this volume of agar will be able to inactivate approximately $\mathbf{0 . 4 2} \mathrm{u}$. penicillin/hr. Calculation of the amounts of penicillin which would enter such a ring from a bead at the centre containing a solution of penicillin $16 \mathrm{u} . / \mathrm{ml}$. showed them to be as follows: after $3 \mathrm{hr}$., nil; after $7 \mathrm{hr}$., $\mathbf{0 . 0 1 7 4} \mathrm{u}$.; after $11 \mathrm{hr} ., 0 \cdot 067 \mathrm{u}$, ; after $20 \mathrm{hr} ., 0 \cdot 19 \mathrm{u}$. Even if the penicillinase were considerably less active at lower concentrations of penicillin, it would be adequate to inactivate such small amounts.

Although the above considerations suggest that penicillinase production by the test organism will result in sharp zone edges it must be emphasized that only minimal penicillinase production is likely to produce this effect. The Mill Hill strain of $B$. pumilus can be shown to produce more potent or more diffusible penicillinase than the I.C.I. strain, and when it is used the zone edges are somewhat ragged because of small colonies which grow up inside the main zone boundary. Ingram (1951) illustrates well the appearance of scattered colonies inside the inhibition zone with a penicillinase-producing resistant staphylococcus, and remarks that fine crenation of the edge makes it unsuitable for assay work.

\section{Streptomycin assays}

We were unable to demonstrate enzymic inactivation of streptomycin or of dihydrostreptomycin by any of the test organisms. It was known, however, 
from the work of Linz (1949) that Gram-positive organisms adsorb streptomycin, and we therefore studied this adsorption quantitatively. Thick suspensions were prepared of B. subtilis, B. pumilus and of Staph. aureus grown for 16-20 hr. on slopes of assay agar. The suspensions were washed with water, and dried from the frozen state. Varying amounts of the organisms were incubated with varying concentrations of streptomycin in phosphate buffer pH $7 \cdot 8$ for periods ranging from $10 \mathrm{~min}$. to several hours. The organisms were

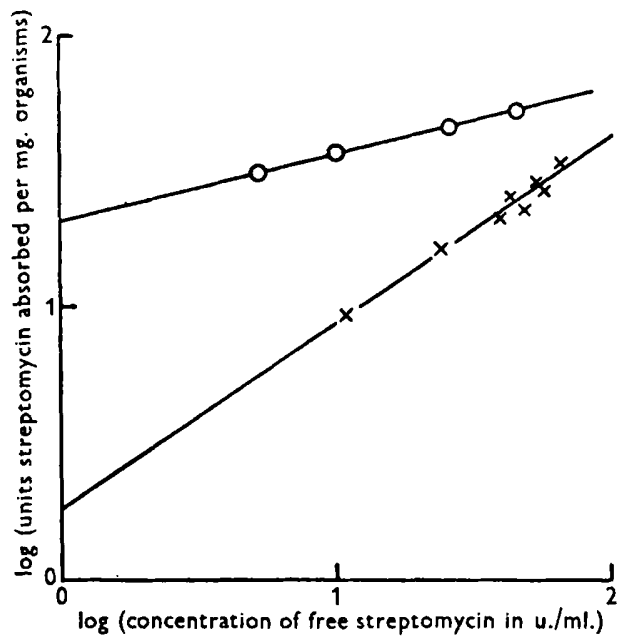

Fig. 7. Relationship between streptomycin adsorbed per mg. dry weight of organisms and concentration of free streptomycin in the medium. Experiments conducted in phosphate buffer pH $7 \cdot 8, \mu=0 \cdot 1$ at $37^{\circ}$. The results are plotted on a logarithmic scale: $\bigcirc-O$, Staph. aureus; $\times-\times$, B. subtilis.

removed by centrifugation and the concentration of streptomycin remaining in the supernatant was estimated biologically (fiducial limits of the assay not more than $\pm 3 \%$ for $P<0.05$ ). It was found that removal of streptomycin by the organisms was independent of time of contact, but that the amount removed per unit weight of organisms depended upon the concentration of streptomycin present. Fig. 7 illustrates the results of experiments with Staph. aureus and $\boldsymbol{B}$. subtilis. The units of streptomycin adsorbed per mg. dry weight of organisms are plotted on a double logarithmic scale against the concentration of streptomycin in $\mathrm{u} . / \mathrm{ml}$. remaining in the supernatant fluid. It will be seen that in both cases the adsorption fits an adsorption isotherm of the Freundlich type. The equations describing their behaviour are: $x=20 \cdot 4 C^{0 \cdot 24}$ for Staph. aureus and $x=1.82 C^{0.68}$ for B. subtilis, where $x=$ units adsorbed $/ \mathrm{mg}$. and $C=\mathrm{u} . / \mathrm{ml}$. in the supernatant. The greater adsorption by Staph. aureus is probably associated with the fact that the suspension used contained much more Gram-positive material than the $B$. subtilis.

Adsorption of streptomycin in this way could account for a sharp fall in concentration at the zone edge, as the following illustrative calculation shows.

Let $\sigma$ be the concentration which would obtain at a given distance from a bead in the absence of adsorption by the organisms, and let $C$ be the actual 
concentration of free streptomycin in the presence of organisms. $(\sigma-C)$ will be the amount of streptomycin adsorbed by the organisms present in $1 \mathrm{ml}$. of the agar layer.

The organisms present in $1 \mathrm{ml}$. after a time $t \mathrm{hr}$. will be $q \cdot \mathbf{2}^{\left(t-t^{2}\right) / \sigma}$, where $q=$ initial inoculum in $\mathrm{mg} . / \mathrm{ml}$, $t^{1}=\mathrm{lag}$ period, $g=$ mean generation time. From the adsorption isotherm we have

$$
\frac{\sigma-C^{\prime}}{q \cdot 2^{\left(a-t^{(2)}\right) / g}}=a C^{b}
$$

where $a$ and $b$ are constants. We also have, from the diffusion equation,

Hence

$$
\sigma=\frac{M}{4 \pi} \overline{h D t} \mathrm{e}^{-r^{2} / 4 D t}
$$

From this equation, using the values obtained experimentally, it is possible to calculate the true concentration (' at a distance $r$ from the bead after a given time $t$.

Consider a bead containing streptomycin $80 \mathrm{u} . / \mathrm{ml}$. placed upon a cold seeded agar plate which is then incubated at $37^{\circ}$. We know from experience that the zone diameter will be about $2 \cdot 15 \mathrm{~cm}$. This diameter is, incidentally, almost equal to the calculated value for $D=0.012 \mathrm{~cm} . .^{2} / \mathrm{hr}$., critical time $45 \mathrm{hr}$, , and critical concentration $0.03 \mathrm{u} . / \mathrm{ml}$. Let us consider then the change of actual concentration with time at a distance $r=1.07 \mathrm{~cm}$. from the bead, and substitute the following values in the equation: $M=2$ units (measured), $h=0 \cdot 4 \mathrm{~cm}$. (measured), $D=0.012 \mathrm{~cm} .{ }^{2} / \mathrm{hr}$. (estimate based on measurement at $4^{\circ}$ ), $q=10^{-4} \mathrm{mg}$. (measured), $t^{1}=1 \mathrm{hr}$. (estimated), $g=0 \cdot 4 \mathrm{hr}$. (measured in shaken broth culture), $a=1 \cdot 82$ (measured), $b=0 \cdot 68$ (measured).

Calculations give the following results:

$\begin{array}{ccc}\text { Time } & \begin{array}{c}C=\text { actual } \\ \text { concentration } \\ (\text { u. } / \text { ml. })\end{array} & \begin{array}{c}\sigma=\text { uncorrected } \\ \text { concentration } \\ (\text { u. } / \text { ml. })\end{array} \\ 1 & 0.000000005 & 0.000000005 \\ 2 & 0.00013 & 0.00013 \\ 3 & 0.0041 & 0.0043 \\ 4 & 0.018 & 0.02 \\ 5 & 0.037 & 0.06 \\ 6 & 0.022 & 0.11\end{array}$

After $6 \frac{1}{2}$ hr., which is equivalent to 13 generations, the density of the organisms in the surface layer would be approximately $2 \mathrm{mg}$. $/ \mathrm{ml}$., which is of the order of the maximal growth to be expected, and it is evident that division would cease, and the equation would no longer be applicable. The calculations entail too many approximations to permit exact conclusions, but they show, nevertheless, that once growth begins, at the site which is later to contain the visible zone edge, the adsorption of streptomycin by the organisms already present can, while growth continues, keep the concentration of free streptomycin from significantly exceeding the critical concentration $(0.03 \mathrm{u} . / \mathrm{ml}$.) . In the case of Staph. aureus, which adsorbs streptomycin more powerfully, 
the same argument can be used but the more powerful adsorption will permit the zone diameter to be much smaller - as in practice it is. We have observed, furthermore, that when streptomycin is added to a plate seeded with $B$. subtilis at $38^{\circ}$ after growth of the test organisms has taken place, no macroscopic lysis occurs. Hence it is immaterial, once the visible zone edge is established, how far streptomycin later penetrates beyond it.

\section{DISCUSSION}

To persons experienced in assay procedures many of the conclusions which have been reached above from theoretical reasoning may appear to be matters of common sense. There is some advantage, however, in being able to consider quantitatively some of the factors governing zone diameters. For example it is possible from a knowledge of the zone diameters produced by two different concentrations of a substance, and of the time of diffusion involved, to calculate its diffusion constant. For obvious technical reasons the value obtained in this way may not be very accurate, but it will be obtained with little trouble. It is also an advantage to be aware of the factors concerned in determining the zone edge, since it becomes clear that the ideal of a perfectly sharp zone edge requires either a homogeneous population of test organisms with a sharply defined critical concentration and no tendency to lysis, or the existence of special circumstances such as we have demonstrated in the case of our penicillin and streptomycin assays. In the absence of such circumstances, although of course such devices as layered plates give some improvement, attempts to secure perfect zone edges may lead only to frustration. In the case of aureomycin assays, for example, we have been unable to demonstrate either 'aureomycinase' activity, or adsorption of aureomycin, by our test organisms, and have had to be content with attempts to improve the zone edge by other methods such as the use of a test organism which after growth produces an alkaline reaction, and thereby swings the $\mathrm{pH}$ in a direction away from the optimum for aureomycin (Valentine \& Johns, 1949).

The prediction that the log. dose will be proportional to the square of the zone diameter, and its experimental verification in most of our assays, may appear to be contrary to common experience, particularly since it should apply to punch plate assays also. This expected relationship would of course become invalid if any factor were to affect small and large zones unequally. As may be seen from Fig. 4, after a given period of diffusion the rate of increase of antibiotic concentration within the zone boundary is greater for the larger amount of antibiotic than for the smaller. The effect of production of a small excess of penicillinase at the zone edge, capable of diffusing inwards, would therefore be to decrease the smaller zones more than the large. Although we have not been able to treat this effect mathematically, it is in the direction which would be required to produce a linear relationship between log. dose and zone diameter, which is that commonly assumed and which we also observed.

From a practical point of view, the distinction between a linear and a quadratic relationship between the log. dose and the zone diameter may prove to be unimportant except when great accuracy is sought. As an example 
let us consider an assay in which the mean zone diameter for a given dose is $2.00 \mathrm{~cm}$. and for $4 \times$ the dose $2.50 \mathrm{~cm}$. If the $\log$. dose is proportional to the diameter, the expected mean diameter for $2 \times$ the dose will be $2.25 \mathrm{~cm}$., whereas if the log. dose is proportional to the square of the diameter the expected mean diameter for $2 \times$ the dose will be $2 \cdot 264 \mathrm{~cm}$. In order to distinguish between these two values a variation of $0.014 \mathrm{~cm}$. in the mean diameter for $2 \times$ the dose must be significant-i.e. the coefficient of variation must be less than $0.25 \%$. This condition would require that at least 16 replicates be used at each dose level if a single plate were used, and more if several plates were involved.

\section{REFERENCES}

Compilation of Regulations for Tests and Methods of Assay and Certification of Antibiotic and Antibiotic-containing Drugs (1951). Vol. I. Washington, D.C., U.S.A.: Federal Security Agency, Food and Drug Administration.

Cooper, K. E. \& Woodman, D. (1946). The diffusion of antisepties through agar gels, with special reference to the agar cup assay method of estimating the activity of penicillin. J. Path. Bact. 58, 75.

Friedman, L. \& Kraemer, E. O. (1930). The structure of gelatin gels from studies of diffusion. J. Amer. chem. Soc. 52, 1295.

Ingram, G. I. C. (1951). Formation of clear zones with 'sensitive' and 'resistant' Staphylococcus aureus in penicillin plate assays. J. gen. Microbiol. 5, 22.

International Critical Tables (1929). Vol. v, p. 63. New York: McGraw Hill.

KaNtorowicz, O. (1951). An antibiotic assay tray. J. gen. Microbiol. 5, 357.

Linz, R. (1949). Sur le mécanisme de l'action de la streptomycine. 1. Action de la streptomycine sur les bactéries. Ann. Inst. Pasteur, 76, 250.

March, H. W. \& Weaver, W. (1928). The diffusion problem for a solid in contact with a stirred liquid. Physical Rev. 31, 1072.

Rowley, D., Cooper, P. D., Roberts, P. W. \& Smith, E. L. (1950). The site of action of penicillin. I. Uptake of penicillin on bacteria. Biochem. J. 46, 157.

. Valentine, F. C. O. \& Johns, R. G. S. (1949). A suggested method for the titration of certain antibiotics. Lancet, ii, 1033.

Vesterdal, J. (1947). Studies on the inhibition zones observed in the agar cup method for penicillin assay. Acta path. microbiol. scand. 24, 273. 